\title{
Effects of High Energy Electron Beams on Small Fields by Using TPS
}

\author{
M.I. El Gohary, Kh. M. El Shahat", E.M. Attalla ${ }^{* *}$ and A. El Yassin* \\ Physics Dept., Biophysics Branch, Faculty of Sciences; \\ *Radiation Oncology Dept., Faculty of Medicine, Al Azhar \\ University and ${ }^{* *}$ National Cancer Institute NCI,Faculty of \\ Medicine, Cairo University, Cairo, Egypt.
}

NERGIES of electron beams studied were $6,8,10$, and $15 \mathrm{MeV}$. 1. Small fields from $2 \times 2$ to $4 \times 4 \mathrm{~cm}^{2}$ were obtained from Cerrobend shields (cutout) attached to the bottom face of a $6 \times 6 \mathrm{~cm}^{2}$ electron cone applicator. Measurements were carried out in a solid phantom with a Roos ionization chamber and Kodak X-OmatV films.

For small field this extra shielding will affect the percentage depth dose (PDD) and the output factors due to little of lateral scatter. The change in dose at maximum depth $\left(\mathrm{d}_{\max }\right)$ as well as changes in the PDDs with small field must be accounted for when measuring output factors.

The aim of the present work was to achieve an accurate calculation of dose for small field dimensions and performing this by evaluating the accuracy of planning system calculation. This will be compared with real measurement of dose for same small field dimensions using different detectors.

Practical work was performed in two steps: (1) Determination of same physical factors required for dose estimation measured by Ross ionization chamber and calculated by treatment planning system (TPS) based on the latest technical report series (IAEA TRS-398). (2) Comparison of calculated and measured data was done.

For all values energies of used where the field area of the electron beam becomes smaller this lead to the following: (i) The PDD of maximum dose shifts toward the surface, (ii) The depth of $90 \%$ and $80 \%$, which are common dose for electron energy, becomes smaller, (iii) The surface dose increases due to scattering and (iv) the dose falloff region becomes more gradual.

The output of the electron beam decreases significantly with reduction in electron beam field area as a consequence of mild lateral equilibrium.

It can be concluded by considering the milde difference between the calculated data and measured values by Roos IC from $3 \times 3$ to $10 \times 10 \mathrm{~cm}^{2}$ field dimenions, where as for $2 \times 2 \mathrm{~cm}^{2}$ field dimenion this difference exceeded $40 \%$ for all energy. Radiographic film showed a variation in results according the following: $10 \%$ for $6 \mathrm{MeV}$, less than 
$3 \%$ for $8 \mathrm{MeV}, 20 \%$ for $10 \mathrm{MeV}$ and $30 \%$ for $15 \mathrm{MeV}$ for all field areas. These results smaller TPS calculated values.

Keywords: Radiotherapy (RT), Treatment planning system (TPS), Ionization champers (IC), Radiographic film (rf), Radiotherapy (RT), IAEA TRS-398.

When the field is reduced below that required for lateral scatter equilibrium, the dose rate decreases rapidly. For small fields, the output factor as well as depth dose can be significantly reduced compared with the broad beam distribution. Most electron beam treatments are planned for a single field technique. For a relatively flat and homogeneous block of tissue, the dose distribution can be found by using the appropriate isodose chart ${ }^{(1)}$.

The definition of a small field in radiation dosimetry is currently very subjective. There is no clear consensus definition as to what constitutes a small field. Commonly, a field area of less than $4 \times 4 \mathrm{~cm}^{2}$ is considered outside the conventional treatment field area that needs special attention both in dose measurements and in dose calculations. A more scientific approach is needed to set the criteria which define a small field condition based on the beam energy and the density of the medium ${ }^{(2)}$.

There are essentially three "equilibrium factors" that determine the scale of the field as small field whether it is considered as small field or not:

i. The dimensions of beam some as projected upon the detectors.

ii. Suitable dimension of detector used in measurements.

Iii. Small scattered radiation due to small field dimensions.

Small field dosimetry plays an important role in modem radiotherapy for many reasons. Treatment planning system commissioning requires the input of beam data, specific to the treatment units. This requires the acquisition of depth dose profiles and beam profiles, as introduced in the previous sections ${ }^{(3)}$

The difficulty in achieving accurate small field dose measurements is similar to factors which affect specific measurements in the dosimetry of larger fields (e.g. the steep dose gradients in penumbral regions and the loss of charged particle equilibrium in the build-up region) but they are accentuated. The loss of lateral charged particle equilibrium generally results in a decreased dose at central axis, rather than just at the beam edges and in the buildup regions. In addition, the narrowing of the beam results in a more peaked lateral dose profile, which escalates the requirement for higher spatial resolution not only in the penumbral regions, but also at the central axis ${ }^{(4)}$.

\section{Instrumentations}

Linear accelerator - Elekta model - high energy (dual energies) 6 and

$15 \mathrm{MV}$ photon beam and multi electron energies $(6,8,10$, and $15 \mathrm{MeV})$.

Egypt. J. Biophys. Biomed. Engng. Vol. 12 (2011) 
The treatment planning system used in this work is Precise Plan. Dosimetric instrumentations are the Roos ionization chamber is used as a reference electron chamber. It is recommended by the IAEA for high precision electron dosimetry in radiation therapy. The chamber has a $4 \mathrm{~mm}$ wide guard ring to exclude any perturbation effect even at low electron energies. Farmer Dosimeter model (2570 / 1B (\# 1164)) and Radiographic film model Kodak X-OmatV. Customize block, this material consists of $50 \%$ bismuth, $26.7 \%$ lead, $13.3 \%$ tin and $10.0 \%$ cadmium and solid phantom it is formed of solid slabs that comprise a cubic phantom of $30 \times 30 \times 30 \mathrm{~cm}^{3}$ dimensions.

\section{Method}

Working in Elekta linear accelerator to determine the absorbed dose you should start with mechanical check should be initially followed, to ensure the suitability of the machine to perform the dosimetric measurements. The laser lines compromise the cross wires in the light field area should be checked. The isocentre point for gantry, collimator and couch rotation should be checked to ensure. Then adjust the solid phantom at $100 \mathrm{~cm} \mathrm{SSD}$, and locating the Roos IC at the depth of maximum dose for each energy $(1.5 \mathrm{~cm}$ for $6 \mathrm{MeV}, 2 \mathrm{~cm}$ for 8 $\mathrm{MeV}, 2.4 \mathrm{~cm}$ for $10 \mathrm{MeV}, 3.8 \mathrm{~cm}$ for $15 \mathrm{MeV}$ ), with zero degree gantry angle, zero degree collimator angle and zero degree couch angle according to IAEA protocol (TRS 398).

Measuring pressure and temperature to calculate the factor $\mathrm{K}_{\mathrm{T}, \mathrm{p}}$ which estimate the effect of pressure and temperature on measurement .

When different small fields were irradiated to measure absorbed dose for each field. Results carried out by TPS were compared with practical data of Roos IC.

Estimating the standard film to be the reference dose gradient by irradiating different films to gradual from 20 to 100 monitor units. Where the irradiated film placed in the percpix sheets placed at surface, process the film and draw an isodose curve through which we can determine the absorbed dose for each irradiated film and optical density value can be determined.

When irradiated different fields and determined the absorbed dose for each field then compared the results with the TPS data.

\section{Results and Discussion}

\section{Absolute values of dose measurement by Roos ionization chamber \\ Small field area for $6 \mathrm{MeV}$}

The calculated data of absorbed dose taken by the precise treatment planning system versus the measured data by Ross ionization chamber were represented in Fig.1 . They were used for different dimensions (cons and cutout) at $\mathrm{D}_{\max } 1.5$ $\mathrm{cm}$ with respected to small field dimensions for $6 \mathrm{MeV}$. 


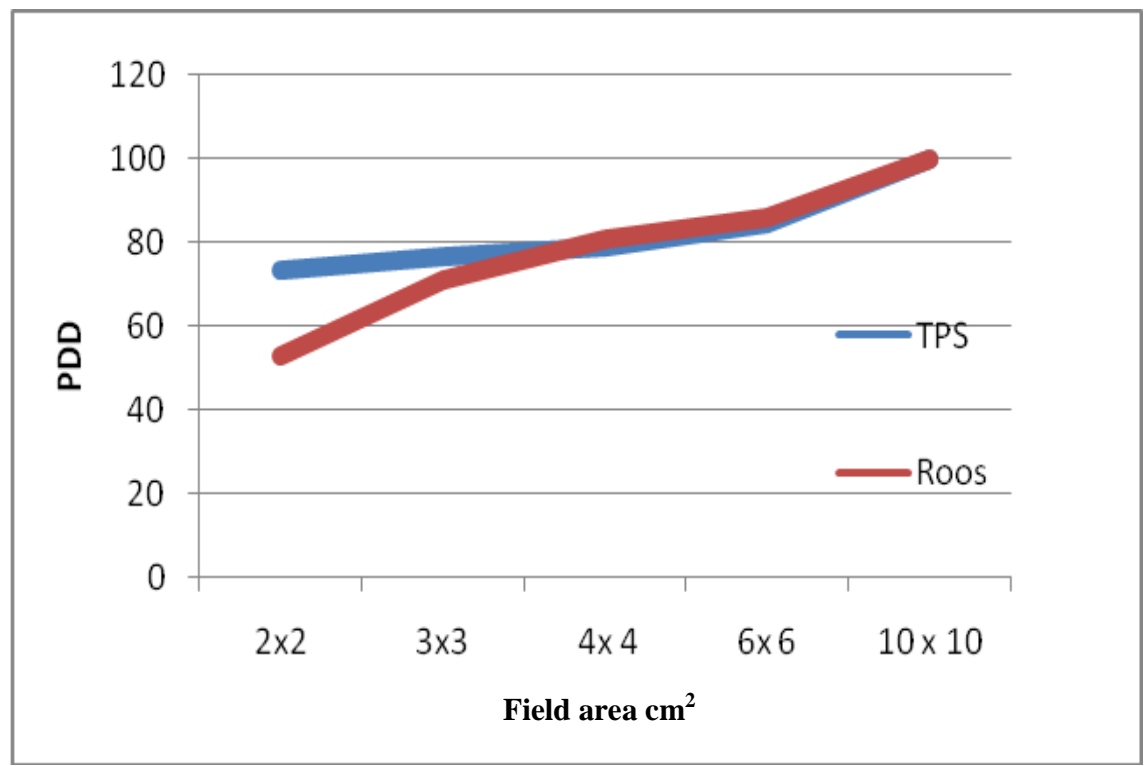

Fig.1. TPS data and measured values by Ross IC for $6 \mathrm{MeV}$.

The discrepancies between calculated data by TPS and measured values by Roos IC were observed in tow regions, First one, the milde difference between the calculated data and measured values from $3 \times 3$ to $10 \times 10 \mathrm{~cm}^{2}$ field dimenions in all energy. Second regoin, the difference between the calculated data and measured values for $2 \times 2 \mathrm{~cm}^{2}$ field dimenions accessed averaged $40 \%$ for all energy.

The volume of measured of Roos chamber very small but pertibration factor is very high for roos due large outside volume incompared with other chamber for this reson roos chamber only for low energies of electrons and determination back scatter radiation ( skin dose $)^{(5,6)}$.

\section{Small field area for $8 \mathrm{MeV}$}

The calculated data of absorbed dose taken by the precise treatment planning system versus the measured data by Ross ionization chamber were represented in Fig.2. They were used for different dimensions (cons and cutout) at $\mathrm{D}_{\max } 2 \mathrm{~cm}$ with respected to small field dimensions for $8 \mathrm{MeV}$. 


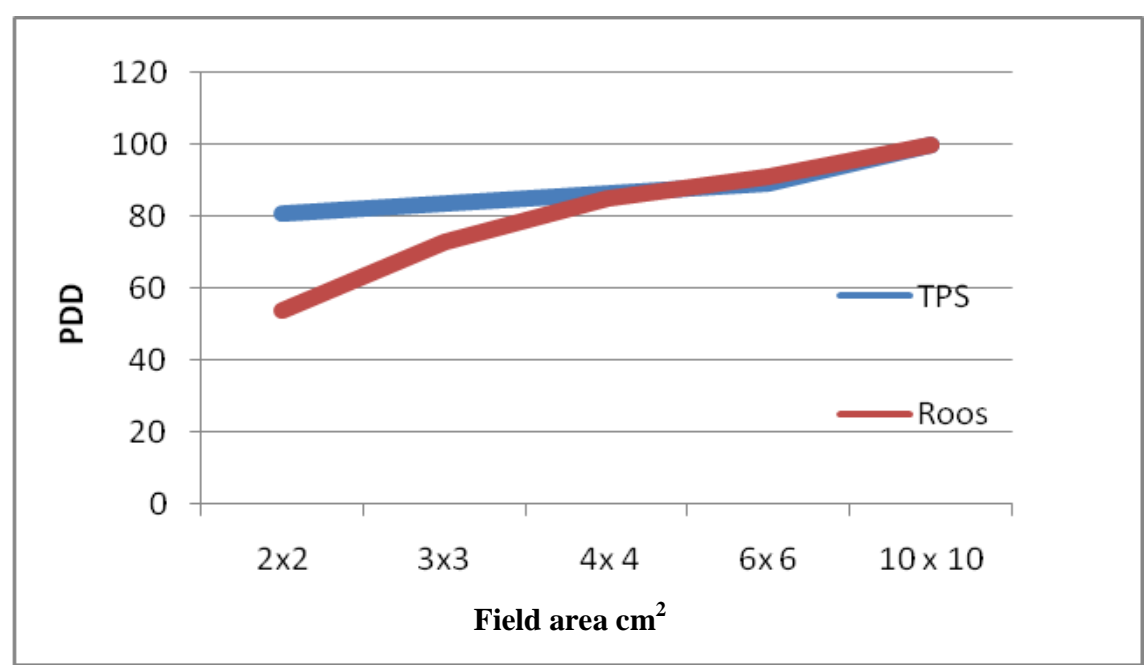

Fig. 2. TPS data and measured values by Ross IC for $8 \mathrm{MeV}$.

Small field area for $10 \mathrm{MeV}$

The calculated data of absorbed dose taken by the precise treatment planning system versus the measured data by Ross ionization chamber were represented in Fig. 3 They were used for different dimensions (cons and cutout) at $D_{\max } 2.4 \mathrm{~cm}$ with respected to small field dimensions for $10 \mathrm{MeV}$.

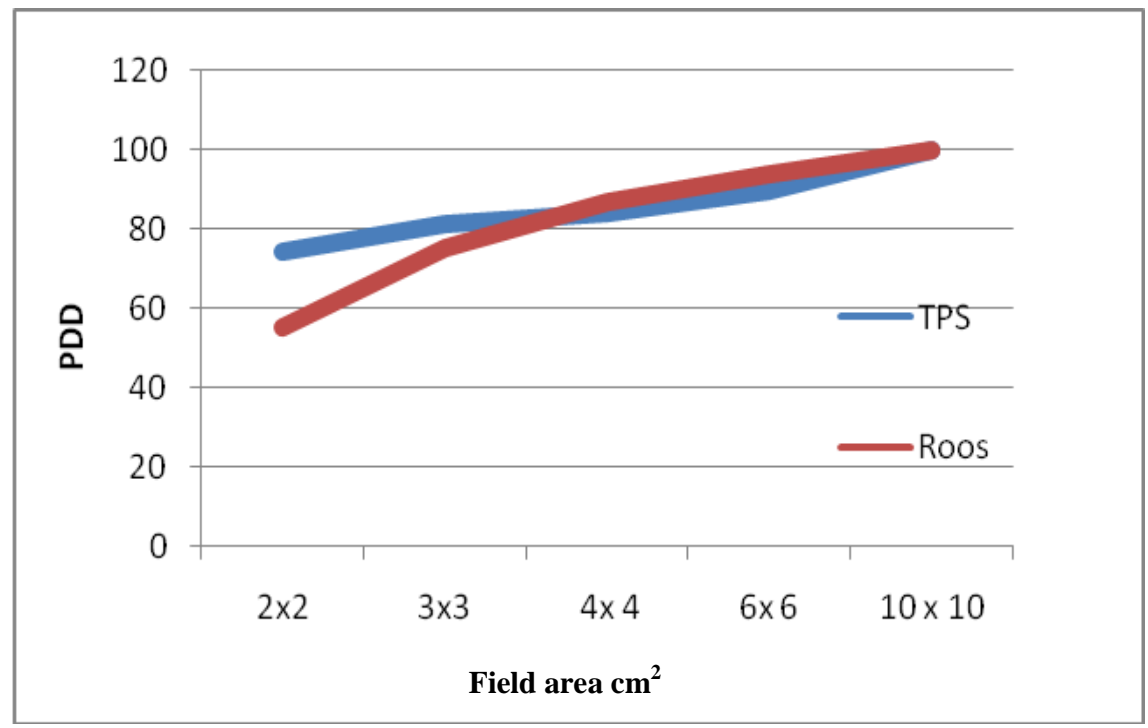

Fig. 3. TPS data and measured values by Ross IC for $10 \mathrm{MeV}$. 


\section{Small field area for $15 \mathrm{MeV}$}

The calculated data of absorbed dose taken by the precise treatment planning system versus the measured data by Ross ionization chamber were represented in Fig. 4. They were used for different dimensions (cons and cutout) at $D_{\max } .8 \mathrm{~cm}$ with respected to small field dimensions for $15 \mathrm{MeV}$.

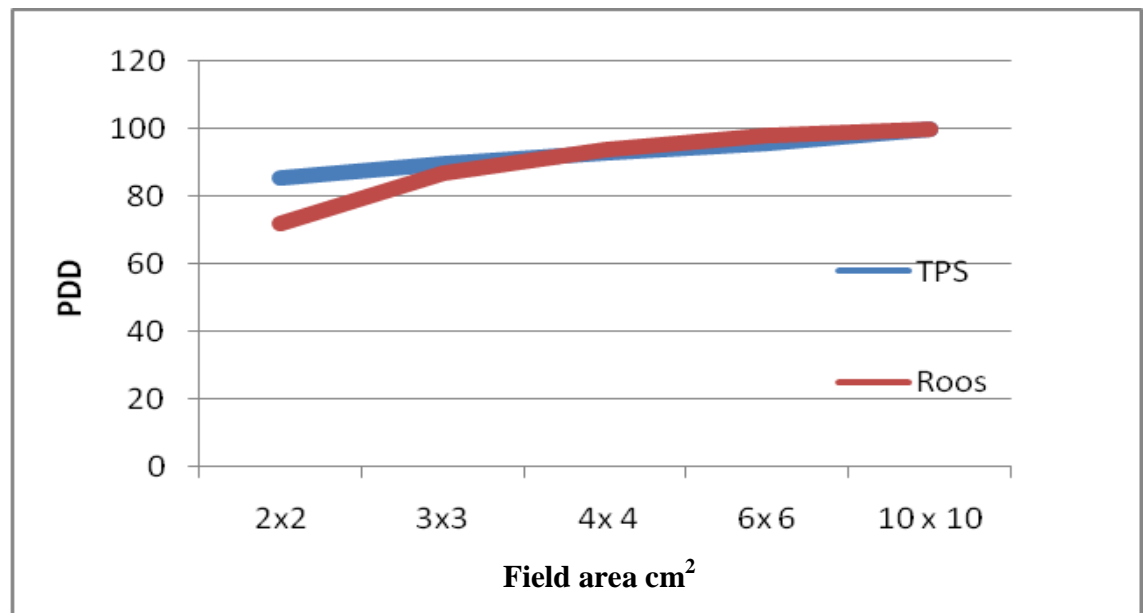

Fig. 4. TPS data and measured values by Ross IC for $15 \mathrm{MeV}$.

Radiographer film for electron beam

Small field area for $6 \mathrm{MeV}$

The calculated data of absorbed dose taken by the precise treatment planning system versus the measured data by radiographic film were represented in the Fig. 5 for different small field dimensions by cutout $\left(2,3\right.$ and $\left.4 \mathrm{~cm}^{2}\right)$ at surface at $50 \mathrm{Mu}$ for $6 \mathrm{MeV}$.

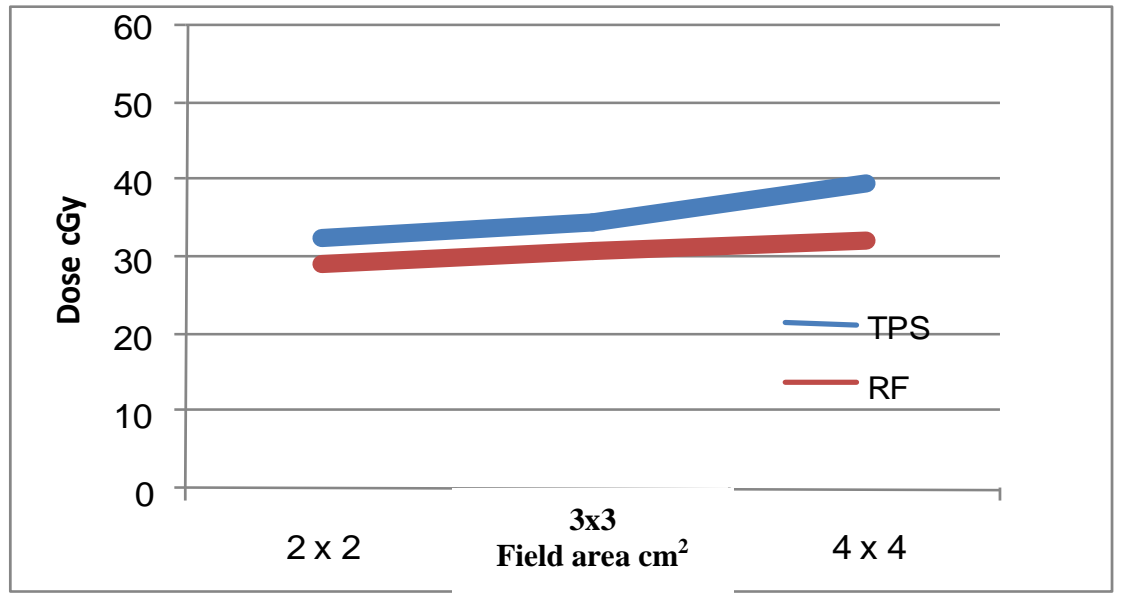

Fig. 5. TPS values against the measured data by radiographic film.

Egypt. J. Biophys. Biomed. Engng. Vol. 12 (2011) 
Figure 5. represents difference between the calculated by TPS and measured data by RF for small field dimensions were averaged to $10 \%$ for all field area. Data variation is agreement with publish by ${ }^{(7)}$ the difference average with 8 to $9 \%$.

\section{Small field area for $8 \mathrm{MeV}$}

The calculated data of absorbed dose taken by the precise treatment planning system versus the measured data by radiographic film were represented in the Fig. 6 for different small field dimensions by cutout $\left(2,3\right.$ and $\left.4 \mathrm{~cm}^{2}\right)$ at surface at $50 \mathrm{Mu}$ for $8 \mathrm{MeV}$.

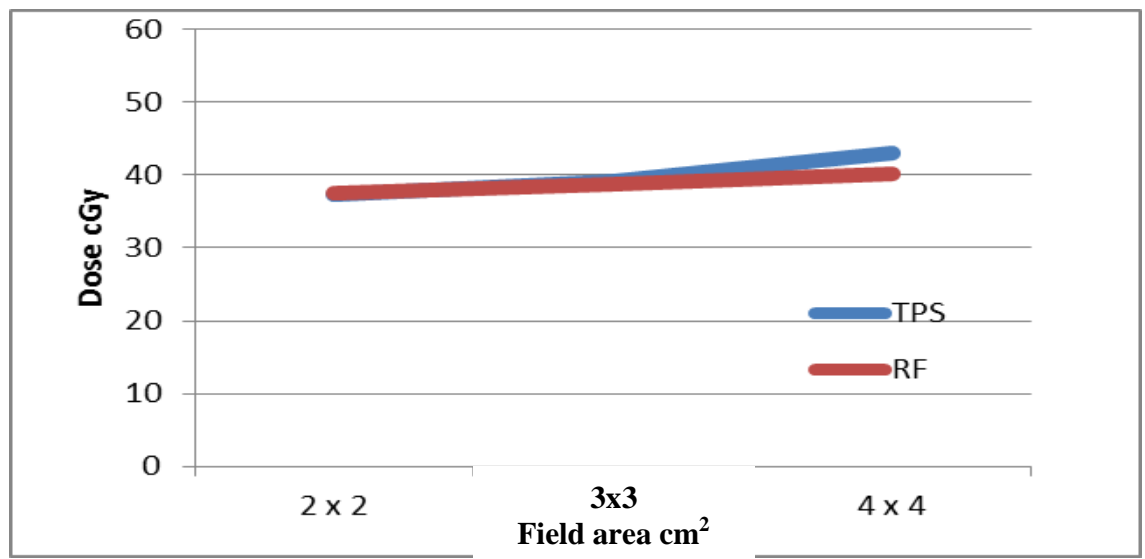

Fig. 6. TPS values against the measured data by radiographic film.

Figure 6. Indicates very mild differences between the TPS and the experimental data for all fields, this difference less than $3 \%$ due to good accuracy of measurement planning system. These data is in agreement with results obtained by (Feliciano Garcia-Vicente, et al $)^{(8)}$, the change within $\pm 1.5 \%$.

\section{Small field area for $10 \mathrm{MeV}$}

The calculated data of absorbed dose taken by the precise treatment planning system versus the measured data by radiographic film were represented in the Fig. 7. for different small field dimensions by cutout $\left(2,3\right.$ and $\left.4 \mathrm{~cm}^{2}\right)$ at surface at $50 \mathrm{Mu}$ for $10 \mathrm{MeV}$. 


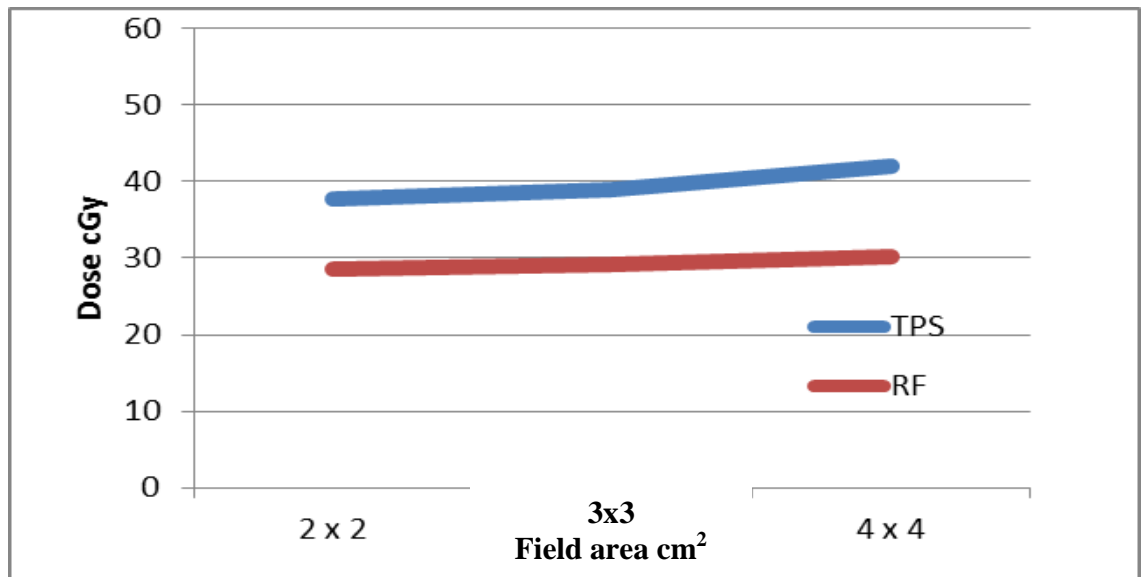

Fig. 7. TPS values against the measured data by radiographic film.

Figure 7. shows difference between the calculated by TPS and measured data by RF for small field dimensions were averaged to $20 \%$ for all field. It indicates large differences between the TPS and the experimental data for all fields. These data is in agreement with results obtained by (Pappas E., Del (2006) ${ }^{(9)}$, the change within $\pm 17 \%$ for electron beams.

\section{Small field area for $15 \mathrm{MeV}$}

The calculated data of absorbed dose taken by the precise treatment planning system versus the measured data by radiographic film were represented in the Fig. 8 for different small field dimensions by cutout $\left(2,3\right.$ and $\left.4 \mathrm{~cm}^{2}\right)$ at surface at $50 \mathrm{Mu}$ for $15 \mathrm{MeV}$.

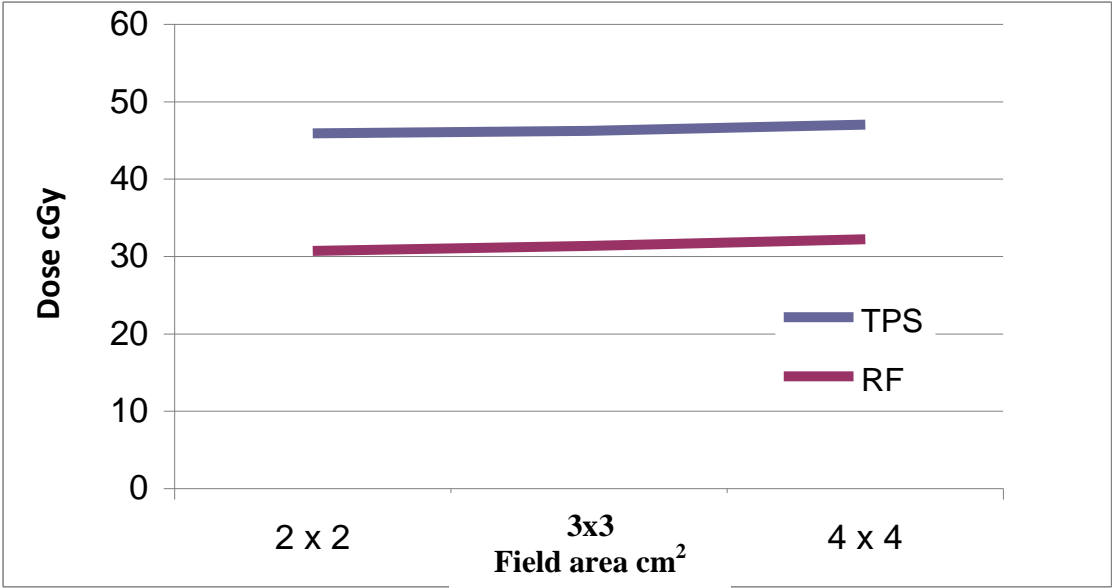

Fig. 8. TPS values against the measured data by radiographic film. 
Figure 8 shows difference between the calculated by TPS and measured data by RF for small field dimensions were averaged to $30 \%$ for all field. It indicates large differences between the TPS and the experimental data for all fields. These data is in agreement with results obtained by Feliciano García-Vicente et al. ${ }^{(8)}$, the change within $\pm 31 \%$ for electron beams.

\section{Conclusion}

- Small field areas for electron beams measured by two detectors Roos IC \& Radiographic film, are dependent on field area and types of detector.

- Results obtained by Roos IC were dependent on field area, due to high value of pertibration factor this lead to an underestimation in absolute dose .

- Results measured by radiographic film were dependent on energy measurment of electron beams. As the energy of electron beam was increased the dose in the build-up region enlarged. Meanwhile underestimation absolute dose of was occurred .

- Patients will benefit from real measurements of TPS system to decrease value of dose delivered to them during radiotherapy procedure.

\section{References}

1. Pappas, E. and Maris, T.G. et al. Experimental determination of the effect of detector size on profile measurements in narrow photon beams. Medical Physics, 33(10), 3700-3701 (2006).

2. Pappas, E. and Petrokokkinos, L. et al. Relative output factor measurements of a 5 mm diameter radiosurgical photon beam using polymer gel dosimetry. Med. Phys. 32(6), 1513-1520 (2005).

3. Zhu, P.A., Jursinic, D.F., Grimm, F., Lopez, Rownd, J.J. and Gillin, M.T. Evaluation of Kodak EDR2 film for dose verification of intensity modulated radiation therapy delivered by a static multileaf collimator X R Medical Physics 29, (8) 16871692 (2002)

4. Esthappan, J., Mutic, S., Harms, W.B., Dempsey, J.F. and Low, D.A., Source Dosimetry of therapeutic photon beams using an extended dose range film Med Phys. 29 (10), 2438-45 (2002)

5. Agostinelli, S., Garelli, S., Piergentili, M. and Foppiano, F., Response to highenergy photons of PTW31014 PinPoint ion chamber with a central aluminum electrode. Med Phys. 35 (7), 3293-3301 (2008)

6. International Atomic Energy Agency (IAEA), Absorbed dose determination in external beam radiotherapy: An international code of practice for dosimetry based on standards of absorbed dose to water, Technical Reports Series, TRS-398, IAEA, Vienna, Austria (2000).

7. Pai, S., Dos, I.J. and Dempsey, J.F., -69., radiographic film for megavoltoge beam dosimetry. Med Phys. 34 (6), 2228-58. (2007)

Egypt. J. Biophys. Biomed. Engng. Vol. 12 (2011) 
8. Feliciano García-Vicente, Cristina Mínguez, Alejandro Floriano, David Sevillano, Leopoldo Pérez, and Juan, J., Study of the uncertainty in the determination of the absorbed dose to water during external beam radiotherapy calibration Pablo Castro, TorresServicio de Oncología Radioterápica, Departamento de Radiofísica, Hospital Universitario "La Princesa," Madrid, Spain Received 23 March 2007; accepted 21 August 2007

9. Pappas, E., Maris, T.G., Papadakis, A., Zacharopoulou, F. and Damilakis, J., Papanikolaou, N. and Gourtsoyiannis, N., Experimental determination of the effect of detector size on profile measurements in narrow photon beams. Med. Phys. 33, 37003710 (2006).

10. Childress, N.L., Dong, L. and Rosen, I.I., Rapid radiographic film calibration for IMRT verification using automated MLC fields. Med Phys. 29 (10), 2384-2390 (2002).

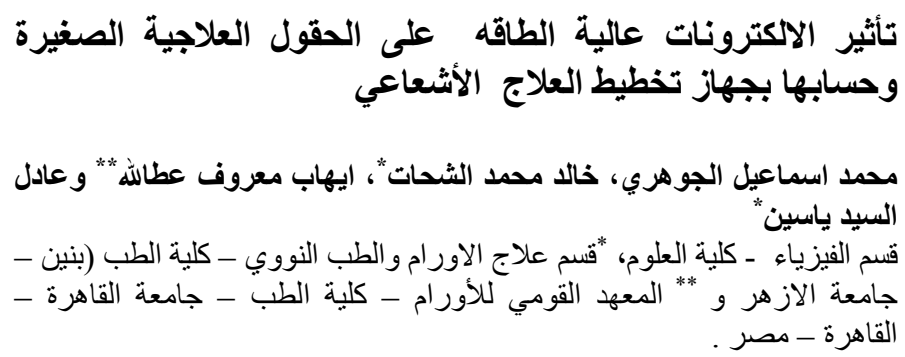

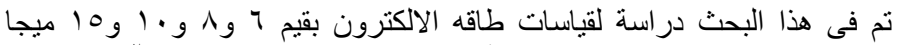

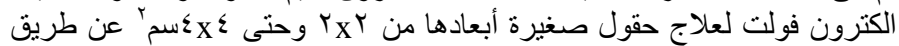

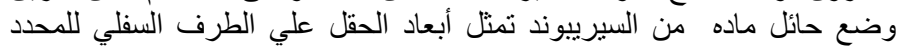

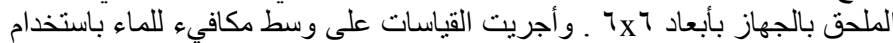

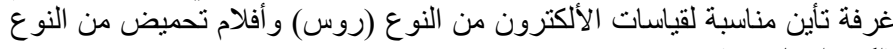

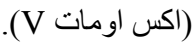

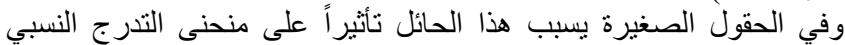
للجر عة مع العمق و على نسبة الأشعاع الصادر من الحقل الخارجي.

وتهدف هذه الدراسة للحصول على حسابات دقيقة للجر عة الممتصة بالحقول

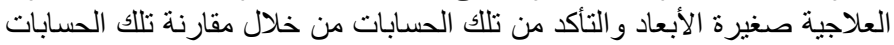

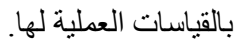

لذلك تمت الدراسة خلال خطوتين الأولي تحديد العوامل الفيزيائية المؤثرة

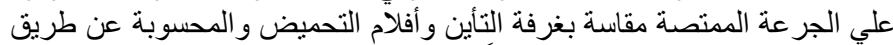

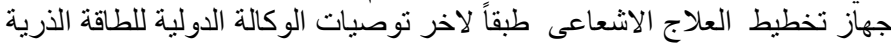

Egypt. J. Biophys. Biomed. Engng. Vol. 12 (2011) 
EFFECTS OF HIGH ENERGY ELECTRON BEAMS...

المنشورة بتقرير القيود العملية والتطبيقية لمعايرة أجهزة العلاج الاشعاعى التماته

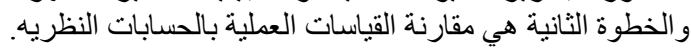

كما أظهرت القياسات أن شعاع الألكترون العلاجي مع أختلاف طاقته يتأثر

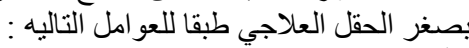

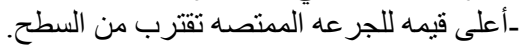

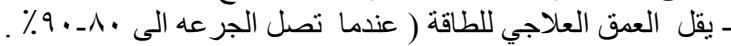

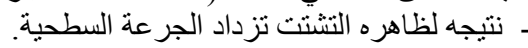

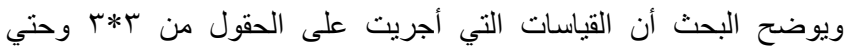

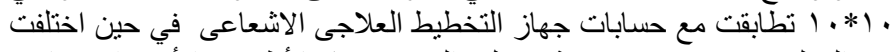

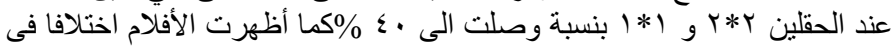

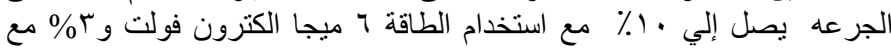

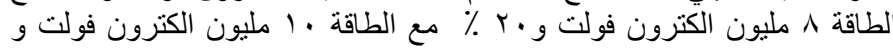

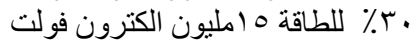

